\title{
Characterization of MIPAS elevation pointing
}

\author{
M. Kiefer ${ }^{1}$, T. von Clarmann ${ }^{1}$, U. Grabowski ${ }^{1}$, M. De Laurentis ${ }^{2}$, R. Mantovani ${ }^{3}$, M. Milz ${ }^{1}$, and M. Ridolfi \\ ${ }^{1}$ Forschungszentrum Karlsruhe, Institut für Meteorologie und Klimaforschung, Karlsruhe, Germany \\ ${ }^{2}$ Operational MIPAS mission planner for ESA, Rhea System SA, Louvain-La-Neuve, Belgium \\ ${ }^{3}$ Responsible for operational MIPAS mission calibration for ESA from 2002 to 2005 - Vitrociset S.p.A., Rome, Italy \\ ${ }^{4}$ Dip.to di Chimica Fisica e Inorganica, Universitá di Bologna, Italy
}

Received: 24 October 2006 - Published in Atmos. Chem. Phys. Discuss.: 13 December 2006

Revised: 14 March 2007 - Accepted: 19 March 2007 - Published: 26 March 2007

\begin{abstract}
Sufficient knowledge of the pointing is essential for analyses of limb emission measurements. The scientific retrieval processor for MIPAS on ENVISAT operated at IMK allows the retrieval of pointing information in terms of tangent altitudes along with temperature. The retrieved tangent altitudes are independent of systematic offsets in the engineering Line-Of-Sight (LOS) information delivered with the ESA Level $1 b$ product. The difference of pointing retrieved from the reprocessed high resolution MIPAS spectra and the engineering pointing information was examined with respect to spatial/temporal behaviour. Among others the following characteristics of MIPAS pointing could be identified: Generally the engineering tangent altitudes are too high by $0-$ $1.8 \mathrm{~km}$ with conspicuous variations in this range over time. Prior to December of 2003 there was a drift of about 50$100 \mathrm{~m} / \mathrm{h}$, which was due to a slow change in the satellite attitude. A correction of this attitude is done twice a day, which leads to discontinuities in the order of $1-1.5 \mathrm{~km}$ in the tangent altitudes. Occasionally discontinuities up to $2.5 \mathrm{~km}$ are found, as already reported from MIPAS and SCIAMACHY observations. After an update of the orbit position software in December 2003 values of drift and jumps are much reduced. There is a systematic difference in the mispointing between the poles which amounts to $1.5-2 \mathrm{~km}$, i.e. there is a conspicuous orbit-periodic feature. The analysis of the correlation between the instrument's viewing angle azimuth and differential mispointing supports the hypotheses that a major part of this latter phenomenon can be attributed to an error in the roll angle of the satellite/instrument system of approximately $42 \mathrm{mdeg}$. One conclusion is that ESA level 2 data should be compared to other data exclusively on tangent pressure levels. Complementary to IMK data, ESA operational LOS calibration results were used to characterize MIPAS pointing. For this purpose MIPAS is used as a ra-
\end{abstract}

Correspondence to: M. Kiefer

(michael.kiefer@imk.fzk.de) diometer while the passage of infrared bright stars through the instrument's field of view is recorded. Deviation from expected time of passage gives information about mispointing. Results are: a pronounced seasonal variation of the LOS is seen before a correction of on-board software took place in December of 2003. Further a pitch bias of 26 mdeg with respect to the platform attitude information is found, which corresponds to $1.45 \mathrm{~km}$ tangent altitude offset towards low altitudes.

\section{Introduction}

\subsection{MIPAS on ENVISAT}

The Michelson Interferometer for Passive Atmospheric Sounding (MIPAS) (Fischer et al., 2000) is a mid infrared Fourier transform spectrometer. It is one of the three atmospheric chemistry instruments onboard the ENVISAT research satellite. ENVISAT is in a sun-synchronous polar orbit, the equator crossings are at 10:00 LT for the descending and at 22:00 LT for the ascending part of the orbit.

MIPAS was operated at a spectral resolution of $0.035 \mathrm{~cm}^{-1}$ until March 2004, when, due to a technical failure, the original measurement mode had to be replaced by a measurement mode of degraded spectral but improved spatial resolution. In its original nominal measurement mode, MIPAS scanned the Earth limb at the 17 tangent altitudes $6,9, \ldots, 39,42,47,52,60,68 \mathrm{~km}$. providing a limb emission spectrum from 685 to $2410 \mathrm{~cm}^{-1}$, including information of at least 30 trace species relevant to atmospheric chemistry and climate change. According to Pellegrini (2003) the pointing accuracy of MIPAS is specified to meet the following criteria: LOS pointing stability (single spectrum) $<0.3 \mathrm{~km} / 4 \mathrm{~s}$, absolute pointing stability $<1.92 \mathrm{~km}$.

Published by Copernicus GmbH on behalf of the European Geosciences Union. 


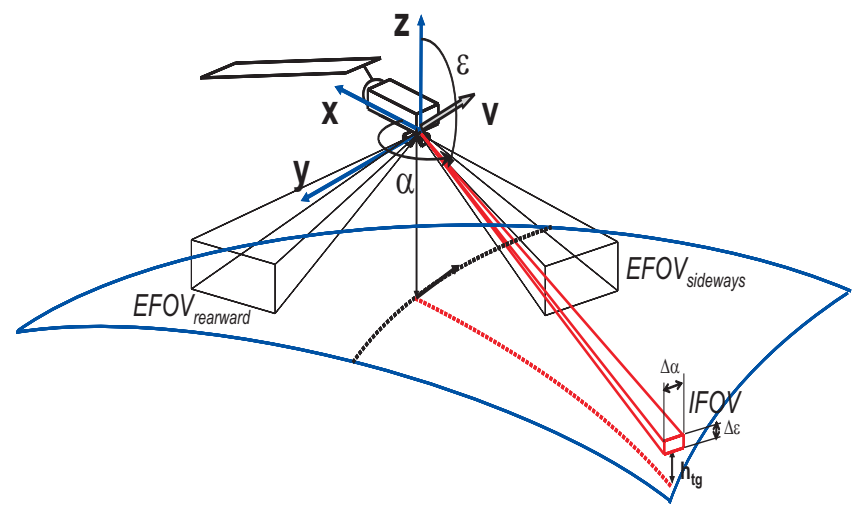

Fig. 1. Setup of MIPAS on ENVISAT. The flight direction of the platform is given by $\boldsymbol{V}$ and the satellite-fixed coordinate system by $\boldsymbol{X}, \boldsymbol{Y}$, and $\boldsymbol{Z}$. The Line-Of-Sight azimuth and elevation are $\alpha$ and $\epsilon$ respectively.

\subsection{Motivation}

Retrieval of atmospheric state variables from limb emission measurements such as MIPAS requires the inverse solution of the radiative transfer equation for the given observation geometry. Obviously, any error in the assumptions on the observation geometry, in particular the tangent altitudes, map directly onto the retrieved state variables. We distinguish between "relative pointing errors", i.e. uncertainties in the altitude differences between adjacent tangent altitudes, and the "absolute pointing errors", which we define are a systematic tangent altitude offset of the entire limb scan. Relative pointing errors cause errors in the retrieved abundances of target species because the difference of the received signals measured at two adjacent tangent altitudes is attributed to an erroneous increment of air number density along the line of sight and an erroneous increment of the length of the ray-path through the atmosphere, which are compensated by erroneous mixing ratios or concentrations of the target species. Where the tangent altitude spacing of nominally $3 \mathrm{~km}$ at $20 \mathrm{~km}$ altitude is overestimated by e.g. $100 \mathrm{~m}$, mixing ratios or concentrations are underestimated by approximately $1.7 \%$, and vice versa. Absolute pointing errors cause an assignment of retrieved mixing ratios to incorrect altitudes, and the related retrieval error is exactly determined by the vertical gradient of the abundance of the target species. In order not to have to rely completely on ENVISAT space craft position and attitude and MIPAS scan mirror position information, methods have been developed to retrieve tangent altitude information directly from the spectra. The absolute pointing information can be retrieved either in terms of pressure at the tangent point (Ridolfi et al., 2000), or in terms of geometrical tangent altitudes (von Clarmann et al., 2003). Both these methods retrieve the relative pointing information in geometric co-ordinates. The different principles behind these approaches, i.e. the tangent pressure retrieval and the retrieval of geometric tangent altitudes are both suitable to avoid the propagation of tangent altitude errors onto the subsequent retrieval of atmospheric state parameters. However, the retrieval of tangent altitudes supports more convenient direct comparison to tangent altitudes inferred from spacecraft position and attitude as well as scan mirror information without the need of an additional hydrostatic interpolation step.

Any independent characterization of the orbit and attitude parameters is not only relevant to the MIPAS instrument but also to the other limb-viewing instruments onboard ENVISAT, GOMOS (Kyrölä et al., 2004; Bertaux et al., 2004) and SCIAMACHY (Bovensmann et al., 1999).

\subsection{MIPAS setup}

MIPAS has been designed to operate in either of two pointing regimes: rearward in the ENVISAT anti-flight direction and sideways in the anti-sun side of the satellite, as illustrated in Fig. 1. The azimuth and elevation angles, $\alpha$ and $\epsilon$ respectively, of the Instantaneous Field-Of-View (IFOV) can move within a maximum allowed range called Extended Field-OfView (EFOV) defined as follows: the azimuth angle can vary from $74.30^{\circ}$ to $110.55^{\circ}$ in the rearward direction and from $159.05^{\circ}$ to $190.56^{\circ}$ in the sideways direction. The elevation angle can vary from $113.06^{\circ}$ to $117.50^{\circ}$ in both directions, with $116.5^{\circ}$ as an angle which represents a middle tangent altitude of nominal limb scans.

Due to this setup, MIPAS pointing is particularly sensitive to the orientation of the satellite $x$ - and $y$-axes (as illustrated in Fig. 1). The instrument, therefore, can be exploited to determine accurate estimates of pitch and roll angles respectively. This can be done in different ways. In this paper we shall present results from two methods. First there is the operational Line-Of-Sight (LOS) calibration by dedicated mesurements as performed by ESA. This method and the corresponding results will be described in detail in Sect. 4. Secondly there is the approach to infer the tangent altitude from a combined temperature/LOS retrieval, as performed at IMK.

\subsection{Retrieval method}

For this study the IMK MIPAS retrieval processor (von Clarmann et al., 2003) is used. MIPAS tangent altitude pointing information is retrieved along with the actual temperature profile from $\mathrm{CO}_{2}$ spectral lines. $\mathrm{CO}_{2}$ is an appropriate tracer for the air mass along the line of sight, since its mixing ratio is sufficiently constant with altitude to trigger a tangent altitude error of no more than $26 \mathrm{~m}$, and its infrared emissions are, contrary to those of $\mathrm{O}_{2}$ or $\mathrm{N}_{2}$, whose altitude distributions are even better known, strong enough for an accurate retrieval. The selection of $\mathrm{CO}_{2}$ transitions, regularization, and numerical representation of retrieved quantities are discussed in detail in von Clarmann et al. (2003), along with a detailed error budget. In this retrieval scheme the tangent altitudes 
are retrieved along with the temperature profile, for a given pressure profile taken from ECMWF. In a second step, during each iteration, the pressure profile is adjusted hydrostatically using the ECMWF pressure at $20 \mathrm{~km}$ altitude, interpolated to the relevant geolocation, as a basis. The $\mathrm{L} 1 \mathrm{~b}$ relative pointing information is used as a constraint in the sense of maximum a posteriori retrieval (Rodgers, 2000). In table 4 of von Clarmann et al. (2003) there are presented the following estimates of the total error of retrieved tangent altitudes: $260 \mathrm{~m}$ below $15 \mathrm{~km}$ altitude, less than $200 \mathrm{~m}$ from $15 \mathrm{~km}$ to $21 \mathrm{~km}, 150 \mathrm{~m}$ or less from $21 \mathrm{~km}$ to $27 \mathrm{~km}$, and then less than $200 \mathrm{~m}$ up to $68 \mathrm{~km}$.

\subsection{Scope of this work}

In Sects. 2-3 we take the engineering tangent altitudes as delivered with the ESA data products as a reference to define mispointing. In Sect. 4 the absolute pointing knowledge is addressed. The engineering tangent altitudes, at the time of the data processing, represent the best knowledge of the tangent altitudes available from inputs partly measured and partly modelled: satellite position and attitude, scan mirror attitude, Earth shape, and atmospheric refraction. It is delivered together with the Level $1 b$ (L1b) and Level 2 (L2) products. L2 products additionally come with a corrected altitude. This is calculated by building up a hydrostatic atmosphere from the retrieved pressure and temperature values, anchored at the lowest engineering tangent altitude (Ridolfi et al., 2000). Clearly the absolute accuracy of this corrected altitude critically depends on the accuracy of the engineering tangent altitude of the lowest tangent point.

The majority of MIPAS data was taken in a measurement mode such that the line of sight (LOS) is essentially backwards with respect to the satellite flight direction (see Fig. 1). In this paper we solely consider data of this backward looking mode. To implement a Sun-synchronous orbit the orbital plane is inclined such that the North Pole is on the right side of the flight path (with respect to flight direction), while the South Pole is on its left side. The LOS azimuth $\alpha$ is changed over the orbit from exactly backwards $\left(=90^{\circ}\right)$ to enhance the coverage of regions beyond the latitude turning points of the orbit. There it is driven up to $110^{\circ}$ in the vicinity of the North Pole while near the South Pole it reaches down to $75^{\circ}$. Between these two extremes there are several discontinuous changes with small steps near the poles and increasingly bigger steps from middle to equatorial latitudes.

In Sects. 2-3 we consider reprocessed data from mid 2002 to 26 March 2004. This is the time range between the first orbit series which covers entire days and the failure of MIPAS's scan mirror mechanism on 26 March 2004. This time period includes 12 December 2003, which corresponds to orbit 9321, the date of the update of the PSO (French acronym for on-orbit position) software. The update was necessary because there was an error in the PSO software leading to a periodic change of the satellite attitude with a period of one year.

It has to be noted that up to now the IMK data processing does by far not cover all available orbits simply due to computing time limitations. Therefore there will be gaps in the data presented in Sects. 2 and 3, which correspond to dates/orbits not yet processed at IMK.

\section{Altitude dependence}

We take as reference the engineering tangent altitude (ETA) $h_{\mathrm{ETA}, i}, i=1 \ldots 17$ as delivered with the L1b product of ESA. The altitude grid is defined as $h_{\mathrm{ETA}, i}=$ $6,9, \ldots, 39,42,47,52,60,68 \mathrm{~km}$, however the lowest tangent altitude, as well as the spacing, unintentionally changes slightly over the orbit. The altitudes set $h_{\mathrm{IMK}, i}, i=$ $n_{\text {low }} \ldots 17$ given by the IMK LOS retrieval results may not always have $n_{\text {low }}=1$, i.e. 17 values because spectra of low tangent altitudes, with features caused by cloud emission, are removed prior to retrieval. Therefore we will not be able to assess $h_{\mathrm{ETA}, i}$ for all altitudes always. At the $i$ th tangent altitude we examine the difference $\Delta h_{i}=h_{\mathrm{IMK}, i}-h_{\mathrm{ETA}, i}$. A value of $\Delta h_{i}$ greater than zero means that the retrieved tangent altitude is larger than the ETA, i.e. the ETA value delivered with the ESA data product gives too low an altitude. In other words $\Delta h_{i}>0$ means that MIPAS was looking higher than indicated by the ETA.

Figure 2 gives an overview over typical features of the height dependence of $\Delta h_{i}$ for a collection of several orbits, where each collection represents approximately one day (which is given in the headline of the panels). Only 13-14 values of $\Delta h_{i}$ are shown in the respective panels, with lowest tangent altitudes in the range $16-18 \mathrm{~km}$. This is because, for the data presented, only altitude levels where $h_{\mathrm{IMK}, i}$ exists for at least $80 \%$ of all geolocations have been considered. Usually in the tropics there are cloud tops as heigh as $15 \mathrm{~km}$. Tangent altitudes below these heights did not enter the LOS retrieval at IMK. So if there are more than $20 \%$ of geolocations contaminated with clouds, there will be no differences shown at all for the respective altitudes. The reason for this rather strict criterion is that, as we shall see in the subsequent sections of this paper, there are features like drifts in time and a dependence of the altitude differences on latitude, which might induce a bias due to sampling (clouds are not evenly distributed along the orbit). There is a change of the height profile of $\Delta h_{i}$-curves with time, where essentially two types can be found: curves of the first type are like those presented in the two upper panels while examples of the second type can be found in the lower panels. The time when the curves change shape is around June of 2003. Before this date they have a slightly wavy appearance with local maxima typically at 27 and $38 \mathrm{~km}$. Afterwards the upper local maximum at $38 \mathrm{~km}$ remains while the lower is shifted downwards to approximately $23 \mathrm{~km}$. Though the main difference is that 

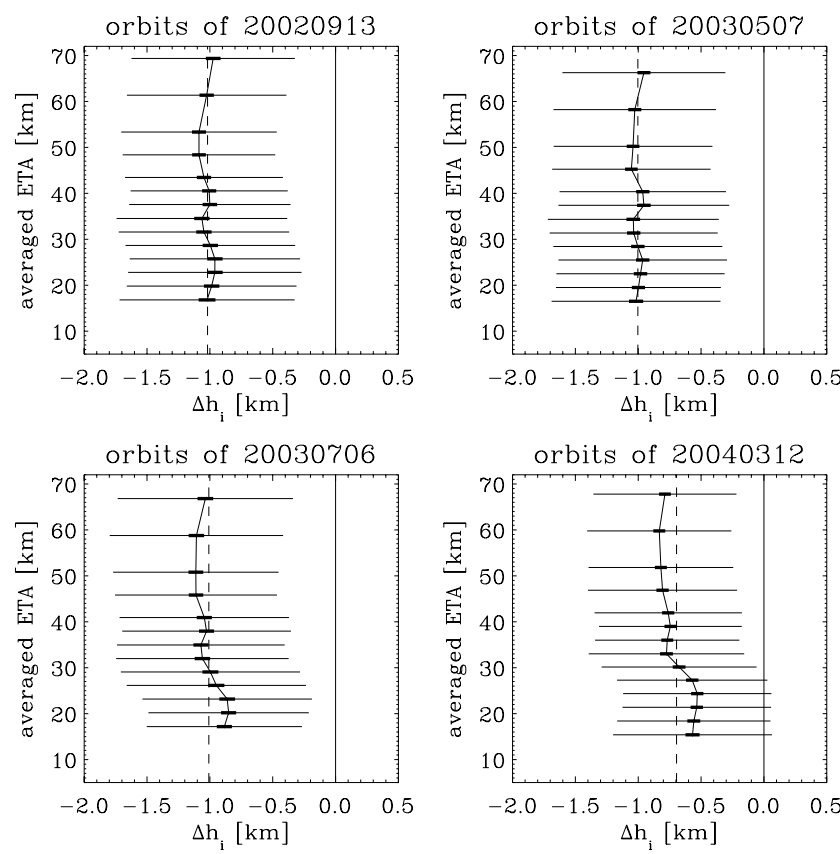

Fig. 2. Altitude dependence of $\Delta h_{i}$ for all orbits of the four days indicated in the panel's headline. Broken lines indicate the average value, thin horizontal lines indicate the standard deviation, and thick horizontal lines give the $99 \%$ confidence limit of the mean value of $\Delta h_{i}$ per altitude.

between the local minimum at $33 \mathrm{~km}$ and the maximum at $23 \mathrm{~km}$ there is an increase in $\Delta h_{i}$ of around $300 \mathrm{~m}$ for the curves of second type while in those of first type it is below $200 \mathrm{~m}$. For the bulk of single geolocations the standard deviation of the single mispointing values per altitude around their mean over altitude lies below $0.2 \mathrm{~km}$. This is not to be mixed up with the standard deviations shown in Fig. 2 which are calculated for 10 successive orbits and therefore contain e.g. drifts and orbit-variations.

The course of $\Delta h_{i}$ with height seems to be quite systematic and hence well characterized by the corresponding standard deviation value. In the remainder of this paper we therefore use the average value at a geolocation

$\Delta h=\frac{1}{17-n_{\text {low }}+1} \sum_{i=n_{\text {low }}}^{17} \Delta h_{i}$.

$\Delta h$ is considered to represent the average difference between the retrieved LOS tangent altitude and the engineering tangent altitude per geolocation to an accuracy given by the rmsvalue. Again $\Delta h>0$ for a geolocation means that on average MIPAS was looking higher than indicated by the engineering tangent altitudes.
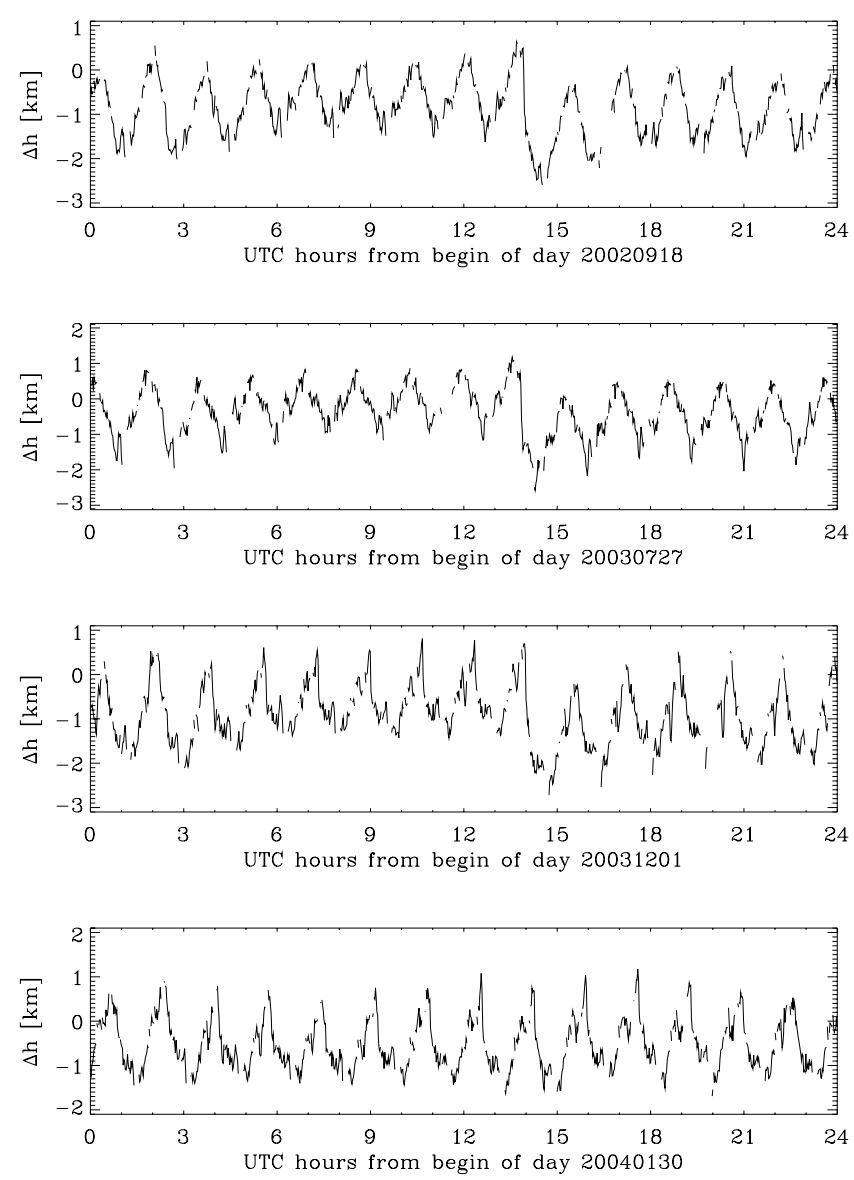

Fig. 3. $\Delta h$ in the course of a day for four different dates. The upper three panels represent three dates before the major update of the PSO-algorithm software on 12 December 2003, while the lowest panel shows a date two weeks after the update.

\section{Time dependence}

Figure 3 shows the values of $\Delta h$ in the course of a day for four different dates. Besides a periodic variation there is a jump at around 14:00-15:00 UTC discernible in the upper three panels of the figure. This type of jump already has been found by von Clarmann et al. (2003) for orbit 2083 and by Kaiser et al. (2004) for orbit 2999. The cause of the jump is an update procedure for the onboard parameters of the platform's attitude, followed by an instant attitude change. This update procedure is performed twice a day at approximately 02:00 UTC and 14:00 UTC. In the vast majority of the data examined only the update procedure at 14:00 UTC shows an effect on $\Delta h$. The update at 02:00 UTC is mostly perceptible as a slightly enhanced amplitude of the corresponding orbit variation. The amplitudes of $2.5-3 \mathrm{~km}$ found by von Clarmann et al. (2003) and Kaiser et al. (2004) for the 14:00 UTC jump are rather exceptional. Usually the jump amplitude at 14:00 UTC is approximately $1-1.5 \mathrm{~km}$. The fact that the 

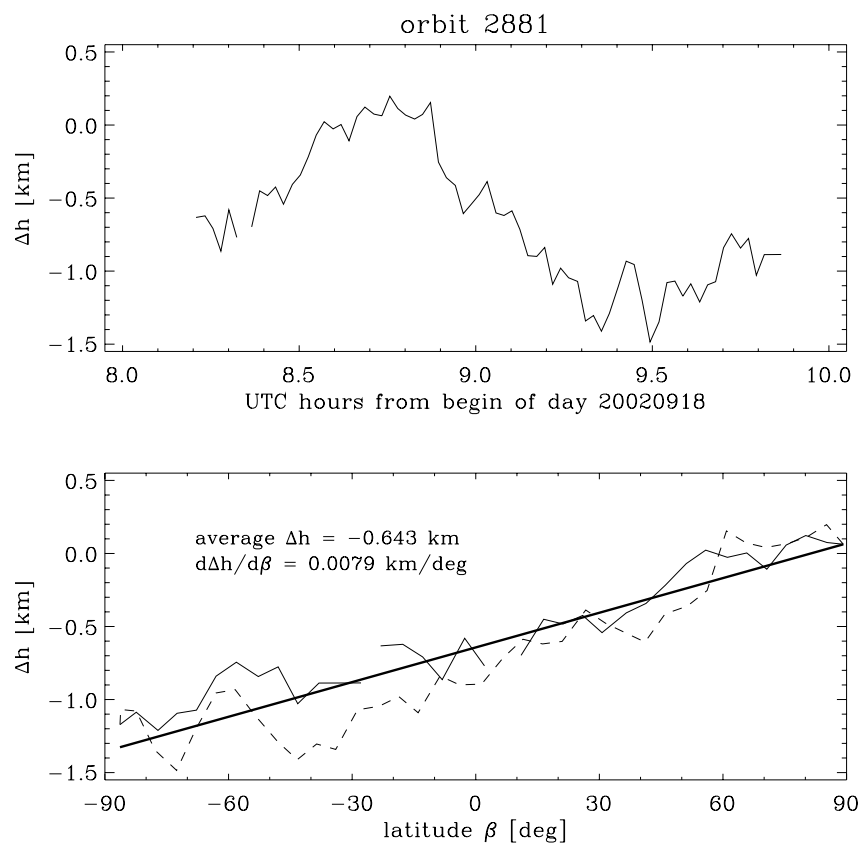

Fig. 4. $\Delta h$ of orbit 2881 plotted against UTC (upper panel) and against geographical latitude $\beta$ (lower panel). In the lower panel the thin solid and broken lines represent values of $\Delta h$ for the ascending and descending part of the orbit, respectively. The thick solid line is the line fitted to the data. The two parameters determining the line are given in the plot frame as average $\Delta h$, i.e. the value at the equator, and $\mathrm{d} \Delta h / \mathrm{d} \beta$, i.e. the slope or gradient.

jump at 02:00 UTC is virtually absent, leads to a rough estimate for the linear trend of $\Delta h$ over a day of $42-63 \mathrm{~m} / \mathrm{h}$ or 70-107 m/orbit. In December 2003 there was a major update of the PSO which largely reduced the jump amplitude. The impact of the gain achieved is illustrated in the lowermost panel of Fig. 3.

There is a pronounced oscillation of $\Delta h$ with a period which corresponds to one orbit, and an amplitude of approximately $1 \mathrm{~km}$. This oscillation persists throughout the whole time span covered by the reprocessed MIPAS off-line data.

\subsection{One orbit}

In Fig. 4 there is shown $\Delta h$ over one orbit. Clearly the extrema of the periodic variation are located at the poles. The course of $\Delta h$ with latitude (lower panel of figure) can roughly be approximated by a line. Therefore a corresponding line fit was performed for all orbits considered henceforth. This fitted line is determined by an offset, i.e. the value at the equator, and a gradient, which is the slope of the line. These two quanitities related to one orbit, offset and gradient, will be examined further.

Figure 5 shows histograms of offset values where only data before the major software update in December 2003 have been considered. In what follows two values character-
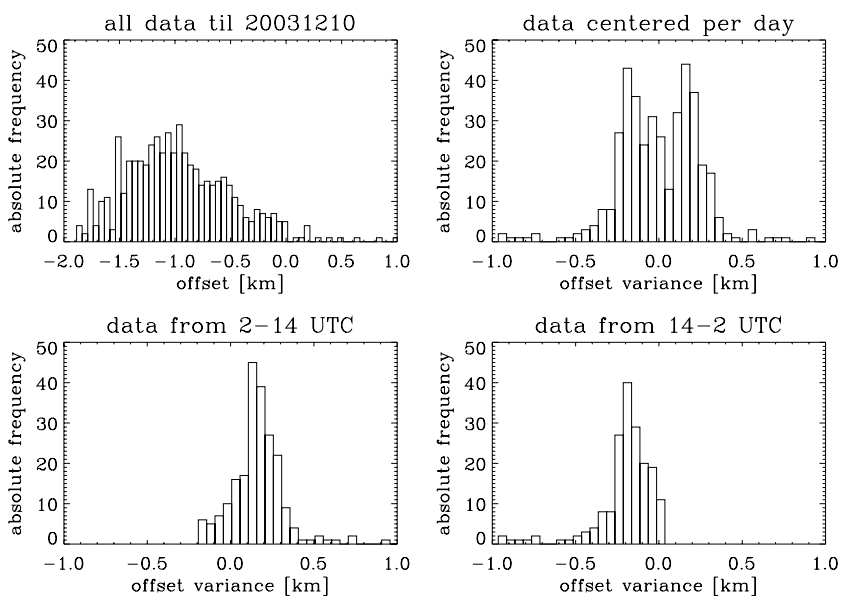

Fig. 5. Histograms of the offset of $\Delta h$. Top left panel: All data before 10 December 2003 (i.e. before the major software update). Top right panel: Data centered on a daily basis (mean value becomes zero). Lower two panels show the data centered per day for offset values of orbits between 02:00 UTC and 14:00 UTC (left) and between 14:00 UTC and 02:00 UTC (right). Binning is equal in all panels.

izing the center of the histograms, namely arithmetic mean and median, will be given in the form mean/median. In the top left panel of Fig. 5 a histogram of all data is shown. The distribution is quite broad with its center at approximately $-0.97 /-1.02 \mathrm{~km}$. For the three other panels all data from a 1.5 $\mathrm{h}$ time window around 02:00 UTC and 14:00 UTC have been removed to avoid unwanted effects of the jumps discussed above. Additionally the data have been centered per day. This means that the daily average value of the offset was subtracted from all single offset values of that day. Hence this procedure gives the variation of the offset around the daily mean value. In the top right panel the corresponding histogram is depicted, the centering on a daily basis of course shifts the distributions such that it is centered around zero. Clearly a double peaked distribution can be seen with the peaks approximately $350 \mathrm{~m}$ apart. If there was only a linear drift, as assumed in the preceding paragraph, one would expect a distribution with a rather flat top. The two lower panels separate the contributors of the two respective peaks. Data from 02:00-14:00 UTC (lower left panel) has a distribution which is centered at $0.17 / 0.16 \mathrm{~km}$, while the distribution of data from 14:00-02:00 UTC is centered at $-0.20 /-0.18 \mathrm{~km}$.

For the sake of completeness we give the corresponding results for data after the 12th of December 2003 (no corresponding plot shown): All data are centered at $-0.55 /-0.56 \mathrm{~km}$, which is a little more than half of the average offset before that date. The data centered per day with removed data points in a $1.5 \mathrm{~h}$ time window around 02:00 UTC and 14:00 UTC are still distributed in a double peaked histogram that can be separated into two single 

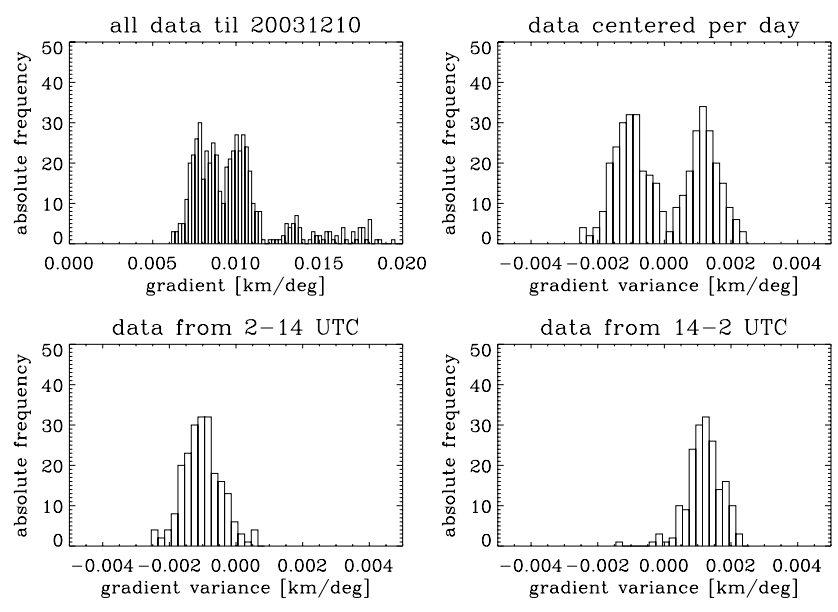

Fig. 6. Histograms of the gradient of $\Delta h$ with respect to latitude. Type of data restrictions as in Fig. 5. Binning is equal in all panels.

peaked ones. Data from 02:00-14:00 UTC is centered at $-0.03 /-0.04 \mathrm{~km}$, and data from 14:00-02:00 UTC at $0.04 / 0.05 \mathrm{~km}$. Hence the peak separation is about a quarter of what it was before the 12 December. Obviously the software update largely improved the pointing of MIPAS with respect to the average offset.

As shown in Fig. 6 the gradient features a behaviour similar to the offset's. The double peaked structure can already be seen in the top left panel, which depicts the histogram of all gradient values of data before the major PSO software update. Arithmetic mean and median of this data set are $0.010 / 0.009 \mathrm{~km} /{ }^{\circ}$. After data from a $1.5 \mathrm{~h}$ time window around 02:00 UTC and 14:00 UTC have been removed, and the procedure of centering on a daily basis has been applied, the two peaks stand out even better separated (top right panel). Again the distribution of values clearly decomposes when split into the classes representing 02:00-14:00 UTC data and 14:00-02:00 UTC data (lower panels). The former is centered at $-0.0010 /-0.0011 \mathrm{~km} /{ }^{\circ}$ while the latter's center is located at $0.0012 / 0.0012 \mathrm{~km} /{ }^{\circ}$. Again an improvement of the data taken after the 12th of December 2003 (not shown as plot) can be observed, however it is much smaller than the offset's: All data is centered at $0.0096 / 0.0097 \mathrm{~km} /{ }^{\circ}$, data from 02:00-14:00 UTC at $-0.0007 /-0.0006 \mathrm{~km} /{ }^{\circ}$, and the 14:00-02:00 UTC data at 0.0009/0.0009 km/ ${ }^{\circ}$.

\subsection{Sucessive orbits}

In Fig. 7 values of $\Delta h$ for several consecutive orbits are shown, separated for ascending and descending parts of the respective orbits. Apart from offset, gradient, drift, and the dependence of the time slots 02:00-14:00 UTC and 14:0002:00 UTC already discussed, there are two additional features standing out clearly. First a difference between the course of $\Delta h$ between ascending and descending parts of
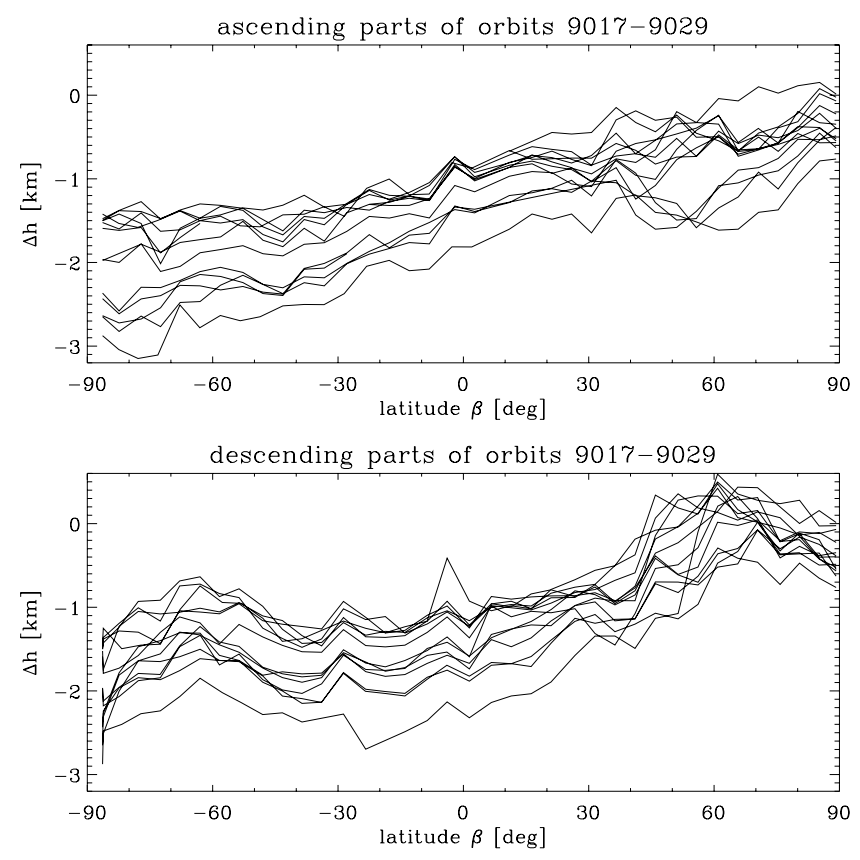

Fig. 7. $\Delta h$ of several orbits of 21 November 2003, separated into ascending (night side, upper panel) and descending (day side, lower panel) parts of the orbits are plotted against geographical latitude.

orbits is discernible. $\Delta h$ of descending orbit parts follows quite well a straight line, while in descending parts it exhibits a wavy appearance. This difference between ascending and descending parts of the orbits is a common feature of all data, while the specific form of the deviation from a straight line of the descending parts is not. The reason for the phenomenon is not clear yet and still under investigation.

Secondly there is an obvious persistence of fine structure in each case in curves of ascending and descending orbit parts. For the orbits shown in Fig. 7 , in $\Delta h$ of ascending orbit parts (upper panel), there is e.g. a marked W-shaped feature between latitudes $-50^{\circ}$ and $-25^{\circ}$, a small positive peak at about $0^{\circ}$, and a small negative peak at about $30^{\circ}$. In descending orbits similar features as e.g. positive peaks at $-30^{\circ}$ and $-5^{\circ}$ and a negative bump around $-40^{\circ}$ can be seen. This persistence of fine structure in $\Delta h$ for successive orbits, bound to certain latitudes, again is a characteristic feature of the whole data set.

\subsection{Correlation of LOS azimuth and $\Delta h$}

In the following we propose a possible reason for the persistent fine structure and the pole-to-pole gradient in $\Delta h$, based on the assumption of an error in the roll angle of the satellite/instrument, which is not accounted for in the data processing. As already described in Sect. 1 the LOS azimuth $\alpha$ of MIPAS is changed periodically over the orbit to enhance the coverage of high latitudes. The adjustment of $\alpha$ is not 
done in a continuous way but rather in steps. From geometric considerations it follows that a deviation of the real from the assumed roll angle of the system, which is not accounted for, will firstly map the orbit periodic adjustment of $\alpha$ into an orbit periodic variation of $\Delta h$ and secondly map the azimuth steps into corresponding discontinuities in $\Delta h$. It has to be noted though, that a change of satellite pitch angle which had an appropriate orbit periodic part would create a very similar behaviour in $\Delta h$ over latitude. Indeed there is an orbit periodic change in the pitch, but its magnitude is much smaller than the effect shown in Fig. 7. This will be discussed in more detail in Sect. 4.2.

Figure 8 gives an overview of the quantities involved. The left column of panels shows $\Delta h$ (top) and LOS $\alpha$ (center) against time, and a scatter plot of both quantities (bottom). In the right column quantities derived from $\Delta h$ and $\alpha$ are depicted. $\Delta(\Delta h)$ is the difference of $\Delta h$ of successive measurement geolocations/times. $\Delta \alpha$ is defined in the same manner as the difference of the LOS azimuth of successive times. $\Delta(\Delta h)$ (top) and $\Delta \alpha$ (center) over time are shown while the bottom panel again contains the corresponding scatter plot. $\Delta h$ plotted against time shows the orbit periodic feature as already discussed in Sect. 3.1. The LOS azimuth $\alpha$ also shows a course periodic in time, with a period of one orbit. Maxima of $\alpha$ of approximately $110^{\circ}$ correspond to maxima in $\Delta h$, i.e. to measurements near the North Pole, while minima of $\alpha$ of around $75^{\circ}$ belong to the southern polar region. $\Delta h$ and $\alpha$ seem to be linearly correlated. The magnitude of the correlation coefficient of 0.78 is well above the $99 \%$ confidence limit. $\Delta(\Delta h)$ still exhibits some periodic feature but a strong noise component, roughly compatible with the value of $0.2 \mathrm{~km}$ given in Sect. 2, is visible. The course of $\Delta \alpha$ over time shows, that the higher the corresponding latitudes are the smaller the changes in the commanded azimuth $\alpha$ become. Around the Equator, which means around $\alpha \approx 90^{\circ}$, the changes are greatest. There seems to be a linear correlation between $\Delta \alpha$ and $\Delta(\Delta h)$ too, with a correlation coefficient of 0.67 . This again is beyond the $99 \%$ confidence limit.

The roll angle $\rho$ can be estimated from a linearized model of the observation geometry (see Appendix) to be

$\rho=\delta_{h, \alpha} \frac{1}{l_{\mathrm{LOS}}}$,

where $\delta_{h, \alpha}$ means either $\mathrm{d} \Delta h / \mathrm{d} \alpha$ or $\mathrm{d} \Delta(\Delta h) / \mathrm{d} \Delta \alpha, \epsilon$ is the LOS elevation angle, and $l_{\text {LOS }}$ means the distance between instrument and tangent point.

The two lowermost plots of Fig. 8 give values of $\delta_{h, \alpha}=0.039 \mathrm{~km} /{ }^{\circ}$ (left) and $\delta_{h, \alpha}=0.046 \mathrm{~km} /{ }^{\circ}$ (right). With $\epsilon \approx 116.5^{\circ}$ and $l_{\mathrm{LOS}} \approx 3200 \mathrm{~km}$ the roll angle for orbits $9027-$ 9028 can be estimated to be approximately in the range of $0.040^{\circ}-0.047^{\circ}$. From the facts that near the North Pole $\Delta h$ is greater than average and that there the azimuth is adjusted to have the LOS more towards the pole, we can infer that the rotation of the instrument/satellite system is counterclockwise
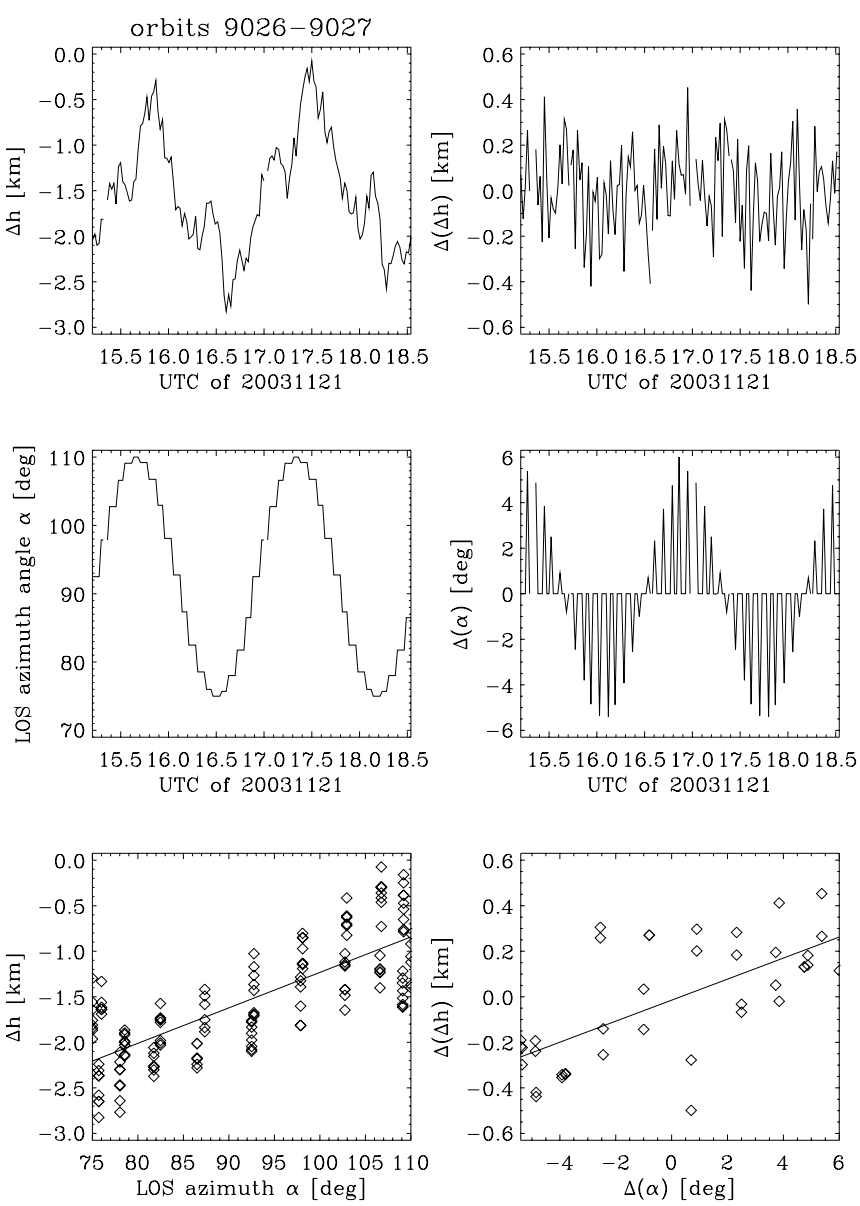

Fig. 8. Correlation of $\Delta h$ and azimuth $\alpha$. Left column shows $\Delta h$ and $\alpha$ against time (top and middle panel, respectively), and the corresponding scatter plot (bottom). Right column has $\Delta(\Delta h)$ and $\Delta \alpha$ against time and the scatter plot of both. Both scatter plots have a fitted straight line overplotted. The definition of the quantities is given in the text.

with respect to the flight direction. In other words, there is a tilt to the left if one looks along the flight path.

A. Dudhia (private communication) reported about retrieval results of observations of May 2005 and December 2006 with MIPAS in the aircraft emission mode. This is a special mode where MIPAS is looking sideways. The respective measurements have been taken with azimuth angles of $\alpha=160^{\circ}$ and $\alpha=190^{\circ}$. The engineering altitudes reported in the L1b data are approximately $5 \mathrm{~km}$ (May 2005) and $4.5 \mathrm{~km}$ (December 2006) too low. This is in rough agreement with our results, both in sign and magnitude, since for $\alpha=180^{\circ}$ we get $\Delta h=2.3 \mathrm{~km}$ for an average a roll angle error of $42 \mathrm{mdeg}$ (see also end of Sect. 3.4). 

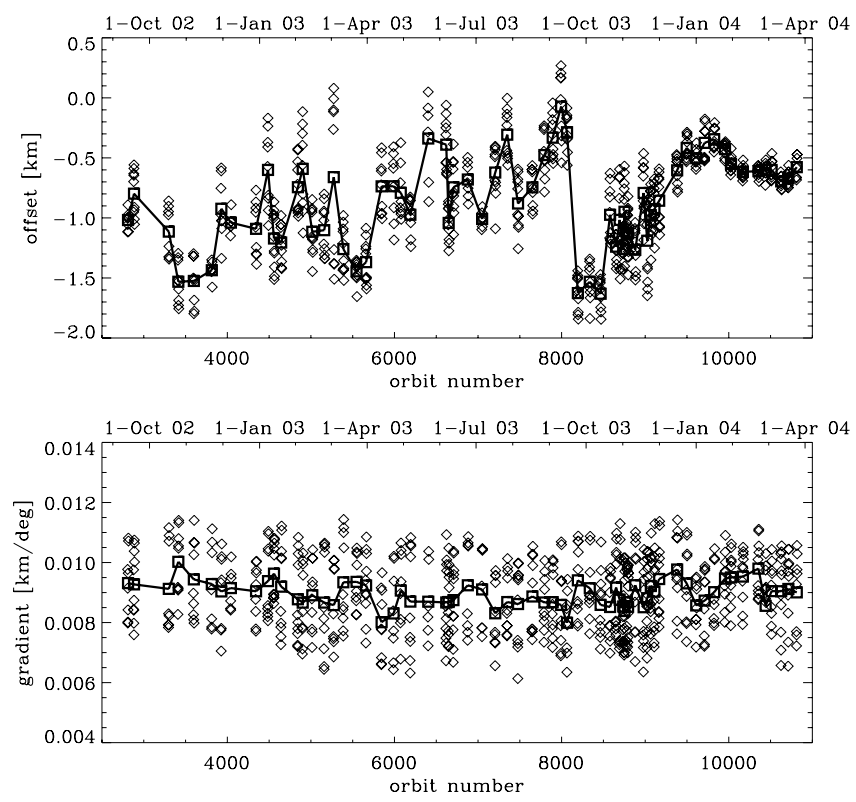

Fig. 9. Offset (top) and gradient (lower panel) drawn over orbit number. Only days with at least 500 processed geolocations have been taken. Each diamond represents a value of a single orbit. The daily average is marked by thick lines connecting squares.

Table 1. Statistics of slopes of fitted lines.

\begin{tabular}{lccc}
\multicolumn{4}{c}{ Statistics of $\mathrm{d} \Delta h / \mathrm{d} \alpha$} \\
\hline time range & mean $\left[\mathrm{km} /{ }^{\circ}\right]$ & std. dev. $\left[\mathrm{km} /{ }^{\circ}\right]$ & median $\left[\mathrm{km} /{ }^{\circ}\right]$ \\
\hline 02:00-14:00 UTC & 0.036 & 0.005 & 0.036 \\
14:00-02:00 UTC & 0.044 & 0.004 & 0.044 \\
00:00-24:00 UTC & 0.040 & 0.004 & 0.040 \\
\hline
\end{tabular}

\begin{tabular}{lccc}
\multicolumn{4}{c}{ Statistics of $\mathrm{d} \Delta(\Delta h) / \mathrm{d} \Delta \alpha$} \\
\hline time range & mean $\left[\mathrm{km} /{ }^{\circ}\right]$ & std. dev. $\left[\mathrm{km} /{ }^{\circ}\right]$ & median $\left[\mathrm{km} /{ }^{\circ}\right]$ \\
\hline 02:00-14:00 UTC & 0.042 & 0.004 & 0.042 \\
14:00-02:00 UTC & 0.042 & 0.007 & 0.043 \\
00:00-24:00 UTC & 0.042 & 0.005 & 0.043 \\
\hline
\end{tabular}

\subsection{Long term behaviour}

To assess the long term trend of MIPAS pointing we again employ the quantities used in the preceding sections. First offset and gradient which characterize the course of $\Delta h$ over an entire orbit are examined. Again data from the time ranges 1:30-3:00 UTC and 13:30-15:00 UTC have been excluded. Figure 9 depicts the corresponding data. The upper panel shows offset values plotted against orbit number, where every diamond represents one orbit. The thick line connecting squares is the daily average. The scatter of the single orbit data is conspicuous, as already visible in Fig. 5. Now it is
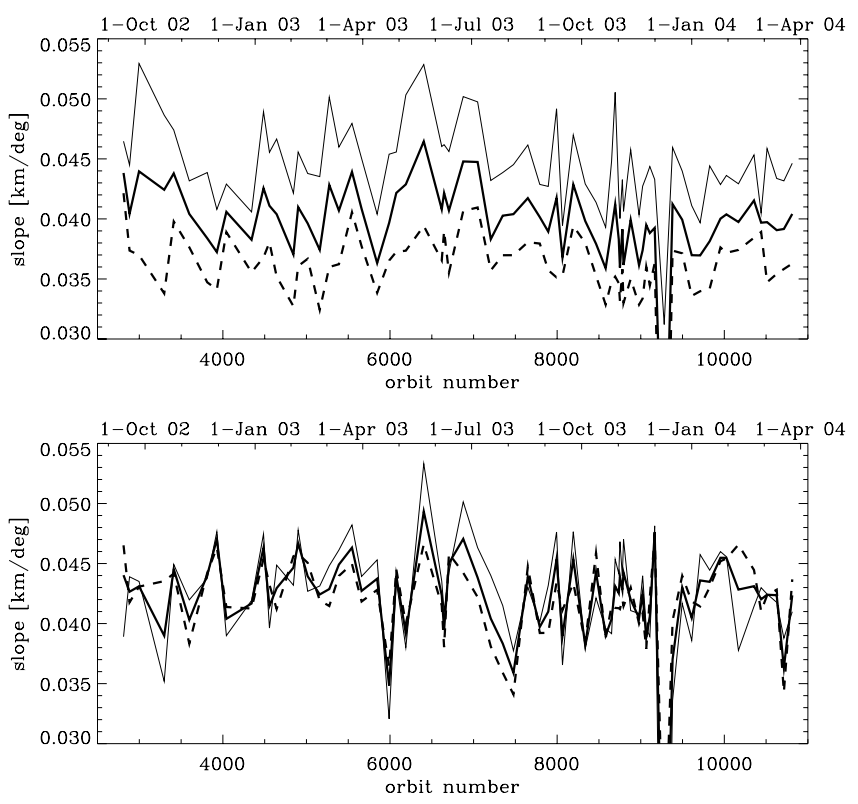

Fig. 10. Slope of the lines fitted to $\Delta h$ and $\alpha$ (upper panel) and to $\Delta(\Delta h)$ and $\Delta \alpha$ (lower panel) plotted over orbit number. Thick solid lines represent the slopes for all data. Thin solid lines give data of the time intervals 14:00-02:00 UTC while thick broken lines mark data of time interval 02:00-14:00 UTC.

obvious that it has two main constituents: Firstly there is the scatter of data of one day which is the manifestation of the daily trend(s) in $\Delta h$, and consequently in the offset, as already discussed in Sect. 3.1. Secondly there is a multitude of trends of different time scales visible, some of which are separated by jumps. A major trend is visible between orbits 3500-8000 (beginning of November 2002 through early September 2003). The daily mean of the offset in this time span changes from -1.3 to $-0.4 \mathrm{~km}$ which corresponds to a trend of $80 \mathrm{~m} / \mathrm{month}$. After a jump of offset values down to $-1.6 \mathrm{~km}$ around orbit 8100 there are two time ranges with quite constant values each, namely $-1.6 \mathrm{~km}$ at orbits 8100 8500 and $-1.1 \mathrm{~km}$ at orbits $8600-9200$. Orbits greater than approximately 9300 (which corresponds to the date of the major software update) show significantly lower scatter in daily offset values as well as a relatively stable average value. This again is a manifestation of the quality gain achieved by the PSO software update. Gradient values (lower panel of Fig. 9) do not exhibit any clear indication of trend while the improvement in data scatter after 12th of December 2003 is small. The details of this have already been discussed at Sect. 3.1.

The slopes of the fitted regression lines of azimuth angle and height offset are plotted over orbit number in Fig. 10. The upper panel shows the slopes of the pair $\Delta h$ and $\alpha$, while in the lower slopes of the $\Delta(\Delta h)-\Delta \alpha$ correlation is drawn. Again only data which does not fall into the two 
time ranges of satellite attitude parameter updates (01:30 3:00 UTC and 13:30-15:00 UTC) has been considered. If there were only the effect of an uncorrected error in the roll angle, both slopes, $\mathrm{d} \Delta h / \mathrm{d} \alpha$ and $\mathrm{d} \Delta(\Delta h) / \mathrm{d} \Delta \alpha$ should have equal values.

As shown in Table 1 this essentially is true. However there is some deviation, which we attribute to to the fact that an uncorrected error in the roll angle might not be the only, albeit greatest, source for the latitude dependence of $\mathrm{d} \Delta h$. Further in $\mathrm{d} \Delta h / \mathrm{d} \alpha$ there obviously is a slight difference between data sets restricted to 02:00-14:00 UTC and to 14:0002:00 UTC. This might be another hint that there are additional effects which contribute to the latitude dependence and that these effects are more pronounced towards the poles, because the calculation of the slopes of fitted lines has different weighting of equatorial an polar points (see Appendix). For the calculation of the roll angle error the value derived from the average of the means of the time intervall $0-24 \mathrm{~h}$ from Table 1 is taken

As a general result we can state that, for the entire time examined here, there is evidence for a roll angle error of the MIPAS measurement geometry of about $42 \pm 5$ mdeg. With respect to the direction of flight the tilt is to the left. The maximum effect of this error in the roll angle occurs in the sideways looking mode, i.e. when the azimuth is $\alpha=180^{\circ}$. In this case a pointing error of $\Delta h=2.3 \pm 0.3 \mathrm{~km}$ would be observed.

\section{MIPAS operational pointing characterization}

\subsection{Method}

As stated in Sect. 1.3, MIPAS pointing is particularly sensitive to the orientation of the satellite $\mathrm{x}$ - and $\mathrm{y}$-axes (see Fig. 1). The instrument, therefore, can be exploited to determine accurate estimates of pitch and roll angles respectively. For this purpose, a dedicated measurement mode called "Line-Of-Sight Calibration Mode" was defined. In this mode the instrument is operated as a radiometer, i.e. the interferometer slides are driven to their end stops and the radiance emitted by bright infrared stars crossing the IFOV is measured.

The trajectory of the stars motion inside the EFOV is dependent upon the viewing direction. While looking in the rearward direction, due to the rotational motion of the satellite in its orbit, the stars have a trajectory approximately parallel to the nadir direction and therefore nearly perpendicular to the XY-plane. In order to acquire Line-Of-Sight (LOS) measurements in the rearward looking geometry, the IFOV is placed at a fixed elevation, near an approaching star and held up until the star has completely crossed the IFOV. The difference between the time at which the star is expected to cross the IFOV and the actual time of crossing is directly re- lated to MIPAS mispointing caused by an error in the pitch angle.

When the instrument looks sideways, the star trajectories parallel to the nadir direction are seen as circular arcs within the EFOV. In this geometry, the IFOV is moved upwards at a pseudo-constant rate in elevation while scanning also in azimuth in order to keep the star azimuth-centred within the IFOV. Differences between actual and predicted star crossing times are again linked to the instrument pointing. Sideways measurements are particularly sensitive to mispointing due to the roll angle of the instrument .

The accuracy of the pointing measurements is strictly related to the accuracy achieved in the determination of the time at which a given star crosses the IFOV. In order to locate the star signal with a good accuracy, the signal-to-noise ratio of the measured radiance has to be increased by observing repeatedly the same star several times and averaging the measured signals. Actually this operation is possible because MIPAS is capable of pointing to the same star for time intervals $40 \mathrm{~s}$ long (in the rearward direction). Therefore, since the nominal star crossing time span is approximately $4 \mathrm{~s}$, the same star can be observed up to ten times in succession.

During the measurements, the signal is acquired only from detectors D1 and D2 (Endemann, 1999) and processed onground using detailed information concerning satellite orbit and platform attitude. Pointing errors are determined by fitting the measurements with a six-parameter model that includes a bias and a sine variation of the pitch and roll error on the platform attitude as a function of the position along the orbit.

LOS measurements cover 2 consecutive orbits and about 60-80 star crossings are observed. The measurements are performed on a weekly basis and processed bi-weekly. This plan allows a proper monitoring of the pointing stability and guarantees the availability of pointing data in case of missing products (unavailability of products containing LOS measurements may be caused by instrument unavailability, failure in commands execution, data transfer problems, failure in data processing, etc.). The baseline for LOS calibration foresees that the absolute bias is compared with the last value disseminated in the ground segment, then a new LOS calibration Auxiliary Data File (ADF) is disseminated only if the absolute difference between the two biases is larger than $8 \mathrm{mdeg}$ (corresponding to about $450 \mathrm{~m}$ in tangent height). The disseminated ADF contains pointing error knowledge to be used within $\mathrm{L} 1 \mathrm{~b}$ data processing to correct pointing during computation of the engineering tangent altitudes.

At the beginning of MIPAS mission, only LOS star measurements from detector D1 were analysed because this detector was less noisy than detector D2. However, starting from September 2003 the noise of detector D1 increased significantly (without impact on science data because this is a low frequency noise) and star signals were no longer visible. Detector D2 is currently used as a backup. However, compared to the beginning of the mission, fewer stars are 


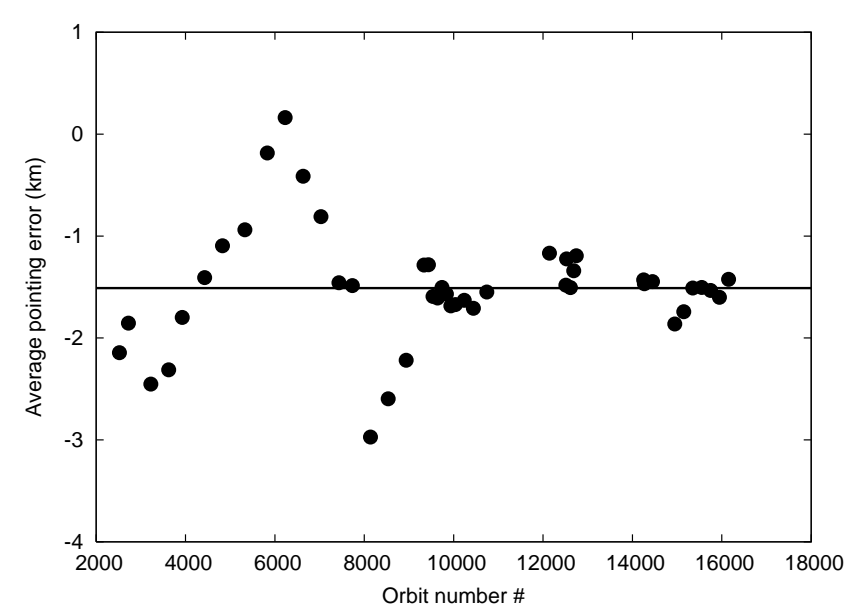

Fig. 11. MIPAS absolute, i.e. uncalibrated, pointing bias as a function of the orbit number, from August 2002 to April 2005. The horizontal solid line represents the average value of the absolute pointing bias. Negative values indicate a bias towards lower altitudes.

observed with good signal-to-noise ratio. To overcome this problem a new commanding scenario has been implemented in November 2004 in order to double the number of observable crossings per star and hence reduce the noise, but no evident improvements have been observed in the noise reduction. Due to the low signal-to-noise ratio of the LOS measurements, it is now hard to determine accurately the orbital variation of MIPAS mispointing. Therefore, the above mentioned fitting procedure is presently used to extract only the bias of pointing errors.

\subsection{Results}

In winter 2004, while investigations on the interferometer mirror drive anomaly were on-going and atmospheric measurements were not possible, the instrument has been exploited to perform an extensive set of LOS measurements. MIPAS LOS data have been inter-compared with attitude information from the ENVISAT star trackers - the so-called restituted attitude information - in preparation for future operational use of restituted attitude in off-line processing (Saavedra et al., 2005). Apart from a pitch offset of $26 \mathrm{mdeg}$, which means that the instrument has a bias of $1.45 \mathrm{~km}$ towards low altitudes, results from the MIPAS LOS campaign agree with the platform star tracker information. Investigations are currently ongoing to find the cause of the observed pitch bias. Moreover, since November 2004, sideways measurements have been interrupted because the related processed data were not reliable. The prototype software for LOS processing is suspected to be responsible for the degradation of the sideways LOS measurements, however investigations on this matter are still in progress.

In Fig. 11 we report the long term trend of MIPAS mis-

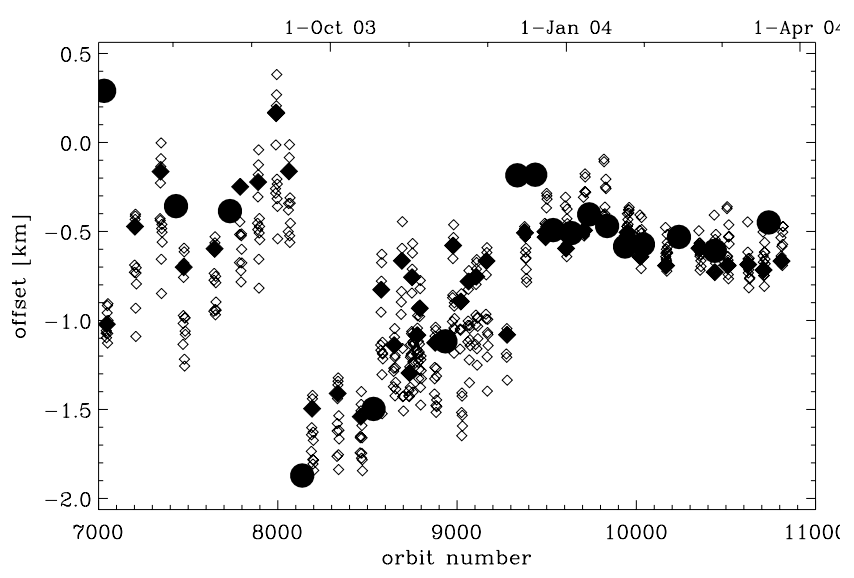

Fig. 12. Comparison of quantities characterizing the mispointing: Big filled circles are the same data as presented in Fig. 11, but shifted $1.1 \mathrm{~km}$ up, while diamonds are the data as shown in the upper panel of Fig. 9. Filled diamonds correspond to orbits which start around 07:00 UTC, the approximate start time of the LOS calibration orbits.

pointing determined during the operational LOS characterization. The figure shows the absolute, i.e. uncalibrated, pointing bias as a function of the orbit number, in the time period from August 2002 to April 2005. Each point is obtained by averaging the values of the pointing error obtained from LOS calibration measurements collected during two full orbits. The conversion of the pointing error from angle (radians) to tangent altitude $(\mathrm{km})$ was again obtained simply multiplying the angles by $l_{\mathrm{LOS}} \approx 3200 \mathrm{~km}$ the average distance between the satellite and the tangent point of typical limb measurements. The pronounced variation of the pointing bias at the beginning of the mission was not related to the MIPAS instrument itself, but to an anomalous behaviour of the attitude of the entire ENVISAT satellite. After the update of the PSO software on 12 December 2003 the amplitude of the variations of the pointing bias was drastically reduced.

Although the results can not be compared directly, because the LOS calibration measurements give absolute pointing deviations, while the engineering altitudes (used to calculate $\Delta h$ ) already contain corrections for mispointing gained by the LOS calibration, it is interesting to see that some features are well visible in both data sets. This is depicted in Fig. 12 which shows data from the jump in mispointing around orbit 8100 until the end of March 2004 (major MIPAS failure) and includes the date of the PSO software update on 12 December 2003. Apart from the fact that the absolute mispointing has been shifted up by $1.1 \mathrm{~km}$, the overall runs of the two data sets do match quite well. Especially after the update (orbits greater than 9321), the coincidence between the two data series is quite good. It is important to note that the difference of $-1.1 \mathrm{~km}$ is no problem of the engineering data, since we compare calibration data with $\Delta h$ which already, via $h_{\text {ETA }}$ 
contains a correction of $-1.45 \mathrm{~km}$. Therefore the actual difference between LOS calibration data and the IMK results is $-1.45-(-1.10) \mathrm{km}$. That means that there are $350 \mathrm{~m}$ missing in the offset correction if one assumes that IMK data were right.

As a further result Saavedra et al. (2005) find that there is an indication for an orbit periodic pitch variation with an amplitude of about 3-4 mdeg. The pitch variation would have an orbit periodic effect in $\Delta h$ of $170-220 \mathrm{~m}$ amplitude, i.e. much less than what is presented in Sect. 3.3. No roll angle effects are considered by Saavedra et al. (2005).

\section{Results of other instruments on board ENVISAT}

\subsection{GOMOS}

The technical note on the ENVISAT Restituted Pitch Assessment by Saavedra et al. (2005) not only deals with dedicated LOS calibration measurements of MIPAS but rather of all limb-viewing instruments onboard ENVISAT to obtain a characterization of the platform's pitch.

The analyses of GOMOS mispointing hint to a orbitperiodic variation of amplitude in the order of some mdeg. This is in accordance with MIPAS results, see Sect. 4.2.

Further there might be an indication of an uncorrected roll angle error. This can be inferred from Fig. 2-1 of Saavedra et al. (2005), where GOMOS elevation mispointing is plotted over azimuth angle. The elevation mispointing clearly decreases from around zero at zero azimuth to $-20 \mathrm{mdeg}$ at $80^{\circ}$ azimuth. Extrapolation to $90^{\circ}$ would give a value of about -25 mdeg which corresponds to a tangent altitude offset of $1.4 \mathrm{~km}$. It has to be noted though, that the course of the dependence of elevation mispointing on azimuth angle does not fit to what would be expected from a roll angle problem. Further investigations on this problem are necessary.

\subsection{SCIAMACHY}

A spatial and temporal characterization of SCIAMACHY limb pointing errors is presented by von Savigny et al. (2005). A specific feature of the tangent height profile of UV radiation is employed to gain information about the true tangent altitudes. Since the method relies on horizontally homogeneous atmospheres, the results are valid only in a latitude band of $\pm 20^{\circ}$ around the equator. The differences of engineering tangent altitudes and retrieved tangent altitudes are averaged over orbits only for these equatorial geolocations. The resulting quantity is called offset. The time span covered is July 2002 through February 2005 with a data gap of 3.5 months in the summer of 2003.

The main results are, that there is a mean offset, a drift, a seasonal variation, and an occurence of two daily jumps. The characteristics of the respective phenomena are different before and after the major update of the PSO-algorithm software on 12 December 2003. While the drift, the amplitude of the seasonal variation, and the magnitude of the 14:00 UTC jump are lower after the software update, the average offset increases as well as the magnitude of the 02:00 UTC jump.

To compare the offset results presented in von Savigny et al. (2005) with $\Delta h$, it is important to note that SCIAMACHY is looking forward with respect to the MIPAS flight path. The definition of the height difference as above gives a reversal of sign compared to our definition. The impact of the two combined facts is that the SCIAMACHY results should be directly comparable to the data presented here, given that both instrument's main source for mispointing is the attitude error of the platform.

The constant offset component seen with SCIAMACHY is $500 \mathrm{~m}$ before and $1 \mathrm{~km}$ after 12 December 2003 . The sign of the change seems to be compatible with our results while, due to the drift and jump features discussed in Sect. 3.4 there is no meaningful average value of the data before the date of the PSO software update.

As already discussed we find a trend of roughly $80 \mathrm{~m} / \mathrm{month}$ between orbits 3500 and 8000 . This corresponds at least in sign to $30 \mathrm{~m} / \mathrm{month}$ reported for the SCIAMACHY data of the time before 12 December 2003, but the time spans used to estimate the trend are very different. As von Savigny et al. (2005) we see that the jump at 14:00 UTC nearly vanishes after this date, however our data basis is not sufficient to either confirm or disclaim their statement that the 02:00 UTC jump has become worse.

\section{Discussion and conclusions}

We have presented a characterization of MIPAS pointing elevation for two quantities which, on different stages of the data processing, represent the knowledge of the instrument pointing. The results of the operational LOS calibration measurements based on star tracking are discussed in Sect. 4, while in Sects. 2-3 results of a LOS retrieval performed at IMK are presented.

We have examined several aspects of the mispointing which is gained as retrieval result from the L1b data. First there is a height dependence of the differences between retrieved tangent altitudes and engineering tangent altitudes. However this height dependence is small, very systematic, and quite stable over a time span of almost two years. Further it is compatible with the estimates of systematic errors which are to be expected for the LOS retrieval (von Clarmann et al., 2003). We take the well defined shape of the height dependence as justification to regard one single value, namely the average of the differences, as a representative quantity for the mispointing at a given geolocation.

Before 12 December 2003 jumps, which occur twice a day, are a regular feature of time series of the mispointing. The first jump at around 02:00 UTC usually is small while the second one at around 14:00 UTC is conspicuous and usually is in the order of $1-1.5 \mathrm{~km}$ but can reach values of $2.5 \mathrm{~km}$. 
The cause for the jumps has been identified by ESA to be the erroneous response of the PSO software to the orbit control information uploaded twice a day. After an update of the PSO software on 12 December 2003 at least the size of the jump at 14:00 UTC is much reduced. Our finding is confirmed by von Savigny et al. (2005), who additionally state that the size of the 02:00 UTC jump has increased after the respective software update. Since the histograms of orbit means of altitude offsets from IMK retrievals are clearly double peaked, the drift between the jumps, which usually has mean values of $70-107 \mathrm{~m} /$ orbit is suspected to be not linear.

The result that there is a strong depence of the mispointing on latitude, together with the observation that there are common small scale features in successive orbits, leads us to suggest that there is a roll angle error in the platform/instrument system which currently is not accounted for. A deviation of the roll angle of $42 \pm 5 \mathrm{mdeg}$ from the assumed value, corresponding to a tilt to the left, referring to the flight direction, would explain a bigger part of the latitudinal behaviour of the mispointing. A confirmation of sign and magnitude of the suggested roll angle error comes from independent retrievals of data with MIPAS in the sideways looking mode (A. Dudhia, private communication). An explanation of the latitude dependence based on an orbit periodic pitch variation alone can be ruled out, since the operational LOS calibrations for MIPAS and GOMOS give consistent values for this effect, which are an order of magnitude below what we actually find. However the particular course of the mispointing on descending orbit parts remains to be explained.

For examination of the longterm behaviour we took the IMK retrieval results as well as dedicated ESA LOS calibration measurements. From IMK data it is found that before the date of the PSO software update the pointing offset shows much scatter: first within one day, which of course is a manifestation of the drift and jumps of the platform, and secondly over a period of about one year with several drift periods separated by jumps. Both types of scatter are very much reduced after the 13 December 2003.

In the MIPAS absolute, i.e. uncalibrated, mispointing determined during the operational LOS characterization, there is a pronounced variation of the pointing bias until the update of the PSO software. This variation is not related to MIPAS, but rather to the attitude of the entire ENVISAT satellite. Again, since 13 December 2003 the amplitude of the variations of the pointing bias was drastically reduced. An average offset of $26 \mathrm{mdeg}(1.45 \mathrm{~km})$ towards low altitudes still remains, though.

If shifted up $1.1 \mathrm{~km}$ the LOS calibration data is well comparable to IMK mispointing results. The difference between LOS calibration data and the IMK results is $350 \mathrm{~m}$. A very small contribution to the difference might be due to the misalignment of the MIPAS detectors, since IMK results rely on data of detectors A1 and A2, while LOS calibrations are performed with detectors D1 and D2. According to Kleinert et al. (2007) the fields of view of all detectors agree within
$1.3 \mathrm{mdeg}$. This would give a maximum error of $75 \mathrm{~m}$ in tangent altitude. Even with this additional error, the difference of $350 \mathrm{~m}$ is not fully consistent with the error estimates of IMK data. Investigations of this problem are currently performed.

With respect to the absolute MIPAS pointing we can state, that for the entire period of MIPAS operation the absolute pointing was well within specified absolute pointing stability of $<1.92 \mathrm{~km}$ (note that the strong variation was due to a platform problem). From IMK data we can further deduce that also the engineering tangent altitudes of reprocessed L1b products are within this boundary. No statement about relative pointing stability can be made, because in the IMK LOS retrieval the $\mathrm{L} 1 \mathrm{~b}$ relative pointing information is used as a constraint in the sense of maximum a posteriori retrieval, and therefore the results will not be independent from engineering information.

Some of our results are definitely relevant for the other limb-viewing instruments onboard ENVISAT, GOMOS and SCIAMACHY, others might be, but an independent confirmation has to be given first to make a corresponding claim. Generally a mispointing problem can have two reasons: first a problem related to the satellite attitude and secondly a problem related to a misalignement of the instrument with respect to the platform. The coincidence of mispointing effects of several instruments therefore will indicate that the problem is caused by the platform. On the other hand the absence of coincidence does not mean that there is no platform problem, since the mounting errors of instruments might compensate for platform attitude errors.

Without doubt, the daily platform jumps and the associated drifts affect all instruments. The same is valid for the variation seen in the MIPAS absolute pointing calibration, as again this shows the effect of the platform's attitude error. Whether there is an effect of the roll angle error is not clear. There is some indication that in GOMOS elevation mispointing there might be such an effect, but this has to be investigated more deeply.

Finally it has to be noted that it is impossible to give a general and simple correction scheme for the MIPAS engineering tangent altitudes. Although some of the systematic deviations could be reduced, e.g. the impact of the roll angle error, many others, less well defined ones, can not.

From this it follows, that comparison and validation work which uses MIPAS data should be based only on the tangent pressure values delivered with the ESA L2 products, and avoid tangent altitudes as reference. To avoid confusion: L2 data products generated with the IMK processor are not affected by the detected pointing fluctuations because the retrieved pointing information is used. 


\section{Appendix A}

\section{Derivation of an approximative roll angle equation}

We assume the Earth to be a sphere of radius $r_{\mathrm{E}}$ and hence neglect the change of the local radius. This is justified due to the fact that we consider only small deviations around the position of a given tangent point. Further we neglect any influence of refraction in the Earth's atmosphere. This again is a reasonable assumption for small deviations from a given point. Refraction has a relevant effect only the ray paths of low tangent altitudes, so the altitude average Eq. (1) we use will only be marginally affected.

In Figs. A1 and A2 a sketch of the geometry of MIPAS looking exactly backwards is shown. The satellite looks down into the atmosphere with an elevation angle $\epsilon$ of approximately $116.5^{\circ}$. The axes $\boldsymbol{X}^{\prime}, \boldsymbol{Y}^{\prime}$, and $\boldsymbol{Z}^{\prime}$ are not the same as $\boldsymbol{X}, \boldsymbol{Y}$, and $\boldsymbol{Z}$ in Fig. 1. While the latter give a system fixed to the satellite, the former ones are set in the following way: $\boldsymbol{Z}^{\prime}$ gives the direction from the Earth's center trough the satellite. $Y^{\prime}$ is directed against the direction of flight, given by $\boldsymbol{V}^{\prime}$. $\boldsymbol{X}^{\prime}$ is chosen to complement $\boldsymbol{Z}^{\prime}$ and $\boldsymbol{Y}^{\prime}$ to a righthand coordinate system. Misalignment of the satellite-fixed system with respect to $\boldsymbol{X}^{\prime}, \boldsymbol{Y}^{\prime}$, and $\boldsymbol{Z}^{\prime}$ can be expressed by a pitch angle error (rotation around $\boldsymbol{X}$ ), a roll angle error (rotation around $\boldsymbol{Y}$ ), and a yaw angle error (rotation around $\boldsymbol{Z}$ ). The satellite altitude is $h_{\mathrm{S}}$.

To derive an approximate expression of the dependence of the roll angle error from the change of $\Delta h$ with azimuth angle, we need two auxiliary planes. The first one, plane A, is defined to contain the tangent point and to be parallel to the $\boldsymbol{Y}^{\prime}-\boldsymbol{Z}^{\prime}$-plane. Plane B contains the tangent point and the Earth's center and is perpendicular to the LOS. The projection factor from plane A onto plane B is

$$
f_{\mathrm{p}}=\frac{1}{\sin \epsilon}
$$

for components parallel to $\boldsymbol{Z}^{\prime}$. is

The length of the LOS between satellite and tangent point

$$
\begin{aligned}
l_{\mathrm{LOS}} & =\left(h_{\mathrm{S}}+r_{\mathrm{E}}\right) \sin \left(\epsilon-\frac{\pi}{2}\right) \\
& =-\left(h_{\mathrm{S}}+r_{\mathrm{E}}\right) \cos \epsilon .
\end{aligned}
$$

The length of $a$ is

$$
\begin{aligned}
a & =l_{\text {LOS }} \sin \left(\epsilon-\frac{\pi}{2}\right) \\
& =-l_{\text {LOS }} \cos \epsilon
\end{aligned}
$$

and the length of $b$ correspondingly is

$$
\begin{aligned}
b & =l_{\text {LOS }} \cos \left(\epsilon-\frac{\pi}{2}\right) \\
& =l_{\text {LOS }} \sin \epsilon
\end{aligned}
$$

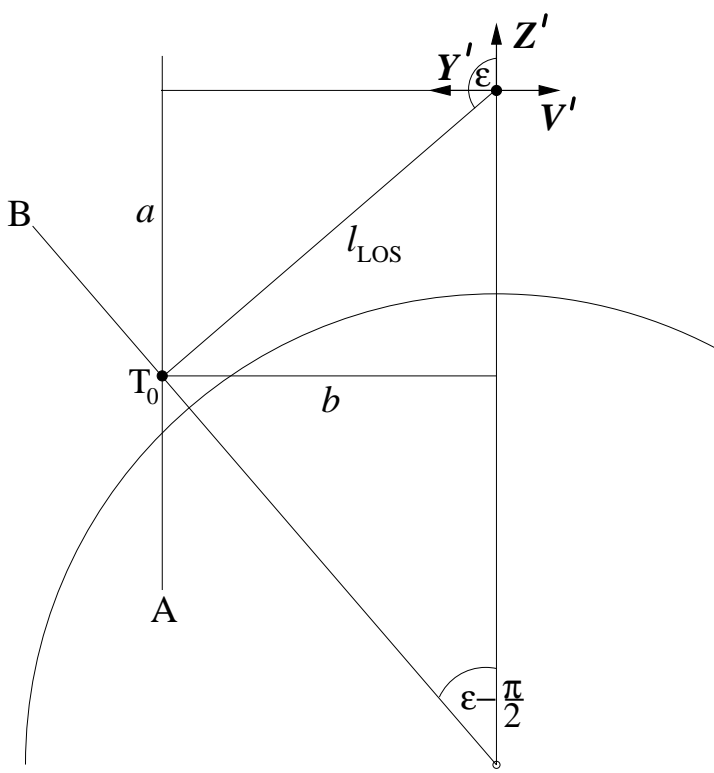

Fig. A1. Viewing geometry of MIPAS as seen from the side; the azimuth angle is $\pi / 2$, i.e. MIPAS is looking exactly backwards. The tangent point for this special case is $\mathrm{T}_{0}$. The definition of planes $\mathrm{A}$ and $\mathrm{B}$ is given in the text.

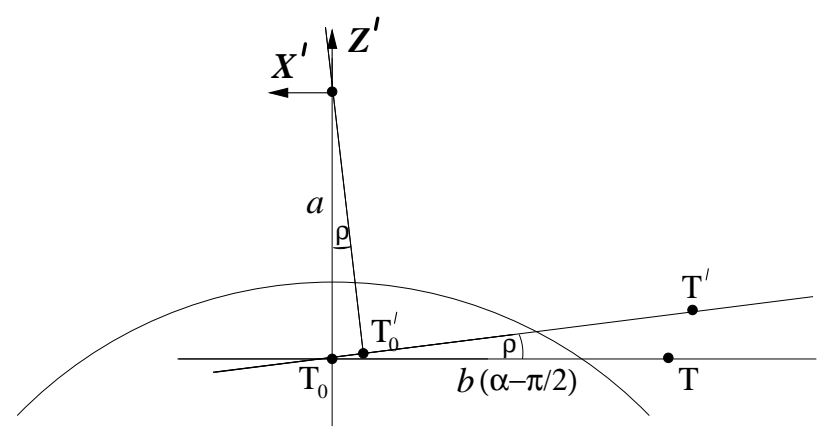

Fig. A2. Viewing geometry of MIPAS for an azimuth angle of $\pi / 2$, i.e. looking exactly backwards, as projected onto the plane A (see Fig. A1 and the text for the plane's definition) and seen in the direction of flight of the satellite. As in Fig. A1 the azimuth angle is $\pi / 2$.

From this and from Fig. A2 it can be estimated that for an azimuth angle of $\pi / 2$ (i.e. MIPAS is looking exactly backwards) the effect of a roll angle error of 50 mdeg would be to shift $\mathrm{T}_{0}^{\prime}$, the true tangent point, $a \rho \approx 1.3 \mathrm{~km}$ sidewards and $a(1-\cos \rho) f_{\mathrm{p}} \approx a\left(\rho^{2} / 2\right) f_{\mathrm{p}} \approx 0.6 \mathrm{~m}$ upwards with respect to the tangent point $\mathrm{T}_{0}$, where the latter number must be increased by $0.15 \mathrm{~m}$ to account for the effect of the Earth's curvature. For the following we assume that there is no yaw angle error and no pitch angle error, but a roll angle error of size $\rho$, and that the angles $\rho$ and $\alpha-\pi / 2$ under consideration are so small that their sines or tangents can be well approximated by the angles themself. For angles less than 
$20^{\circ}$ this is true with an error of below $5 \%$. Then, as can be found from Fig. A2, an azimuth angle $\alpha>\pi / 2$ will cause the true tangent point $T^{\prime}$ to be higher than the targeted tangent point $\mathrm{T}$. If the deviation of the azimuth differs more than $\rho a / b=-\rho \cot \epsilon \approx 13 \mathrm{mdeg}$ from $\pi / 2$, as it actually does in the MIPAS LOS commanding, it is well justified to neglect the tiny and constant contribution which comes from the case $\alpha=\pi / 2$. The resulting difference parallel to $Z^{\prime}$ in the plane A then is $(\alpha-\pi / 2) \rho b$. By projection onto plane B this becomes an altitude difference

$$
\begin{aligned}
\Delta h & =\left(\alpha-\frac{\pi}{2}\right) \rho b f_{\mathrm{p}} \\
& =\left(\alpha-\frac{\pi}{2}\right) \rho l_{\mathrm{LOS}} .
\end{aligned}
$$

Hence

$$
\frac{d \Delta h}{d \alpha}=\rho l_{\mathrm{LOS}}
$$

and therefore

$\rho=\frac{d \Delta h}{d \alpha} \frac{1}{l_{\mathrm{LOS}}}$.

From the assumption of small azimuth angles it becomes clear that our approximation for the roll angle error works best with data corresponding to values of $\alpha$ close to $90^{\circ}$. This is the reason why we believe that the quantity $\mathrm{d} \Delta(\Delta h) / \mathrm{d} \Delta \alpha$ gives more reliable results when used to calculate $\rho$, because the slope of the fit line is determined strongly by the data points, which lay the farthest apart. For $\Delta \alpha$ as abscissa, these indeed are data points which belong to azimuth angles close to $90^{\circ}$, since in the phase of the orbit when the MIPAS LOS has only a small sideways looking component, the changes in azimuth are greatest (see Fig. 8, middle row).

Acknowledgements. We thank the anonymous referees for their very constructive comments which helped us to improve the paper. Anu Dudhia has provided helpful comments on an early draft of the manuscript and supported us with the results of his AE mode retrieval. F. Niro (Serco), and R. Koopman (ESA/ESRIN) have provided data and documents.

Edited by: D. Grainger

\section{References}

Bertaux, J. L., Hauchecorne, A., Dalaudier, F., Cot, C., Kyrölä, E., Fussen, D., Tamminen, J., Leppelmeier, G. W., Sofieva, V., Hassinen, S., Fanton d'Andon, O., Barrot, G., Mangin, A., Théodore, B., Guirlet, M., Korablev, O., Snoeij, P., Koopman, R., and Fraisse, R.: First results on GOMOS/ENVISAT, Adv. Space Res., 33, 1029-1035, 2004.

Bovensmann, H., Burrows, J. P., Buchwitz, M., Frerick, J., Noël, S., Rozanov, V. V., Chance, K. V., and Goede, A. P. H.: SCIAMACHY: Mission objectives and measurement modes, J. Atmos. Sci., 56, 127-150, 1999.
Endemann, M.: MIPAS Instrument Concept and Performance, in Proceedings of European Symposium on Atmospheric Measurements from Space, Noordwijk, Netherlands, 18-22 January, vol. WPP-161, pp. 29-43, European Space Agency, ESTEC, Noordwijk, The Netherlands, 1999.

Fischer, H., Blom, C., Oelhaf, H., Carli, B., Carlotti, M., Delbouille, L., Ehhalt, D., Flaud, J.-M., Isaksen, I., López-Puertas, M., McElroy, C. T., and Zander, R.: Envisat-MIPAS, an instrument for atmospheric chemistry and climate research, European Space Agency-Report SP-1229, C. Readings and R. A. Harris (eds.), ESA Publications Division, ESTEC, P.O. Box 299, 2200 AG Noordwijk, The Netherlands, 2000.

Kaiser, J. W., von Savigny, C., Eichmann, K.-U., Noël, S., Bovensmann, H., and Burrows, J. P.: Satellite-pointing retrieval from atmospheric limb-scattering of solar UV-B radiation, Can. J. Phys., 82, 1041-1052, 2004

Kleinert, A., Aubertin, G., Perron, G., Birk, M., Wagner, G., Hase, F., Nett, H., and Poulin, R.: MIPAS Level 1B algorithms overview: operational processing and characterization, Atmos. Chem. Phys., 7, 1395-1406, 2007

Kyrölä, E., Tamminen, J., Leppelmeier, G. W., Sofieva, V., Hassinen, S., Bertaux, J. L., Hauchecorne, A., Dalaudier, F., Cot, C., Korablev, O., Fanton d'Andon, O., Barrot, G., Mangin, A., Théodore, B., Guirlet, M., Etanchaud, F., Snoeij, P., Koopman, R., Saavedra, L., Fraisse, R., Fussen, D., and Vanhellemont, F.: GOMOS on Envisat: an overview, Adv. Space Res., 33, 1020 1028, 2004.

Pellegrini, A.: Envisat-1 Products Specifications, Volume 12 of MIPAS Products Specifications, PO-RS-MDA-GS-2009, ESA, 2003

Ridolfi, M., Carli, B., Carlotti, M., von Clarmann, T., Dinelli, B., Dudhia, A., Flaud, J.-M., Höpfner, M., Morris, P. E., Raspollini, P., Stiller, G., and Wells, R. J.: Optimized Forward and Retrieval Scheme for MIPAS Near-Real-Time Data Processing, Appl. Opt., 39, 1323-1340, 2000.

Rodgers, C. D.: Inverse Methods for Atmospheric Sounding: Theory and Practice, vol. 2 of Series on Atmospheric, Oceanic and Planetary Physics, edited by: Taylor, F. W., World Scientific, 2000.

Saavedra, L., Mantovani, R., and Dehn, A.: ENVISAT Restituted Pitch Assessment, Technical Note ENVI-SPPA-EOPG-TN-050011, ESA, 2005.

von Clarmann, T., Glatthor, N., Grabowski, U., Höpfner, M., Kellmann, S., Kiefer, M., Linden, A., Mengistu Tsidu, G., Milz, M., Steck, T., Stiller, G. P., Wang, D. Y., Fischer, H., Funke, B., GilLópez, S., and López-Puertas, M.: Retrieval of temperature and tangent altitude pointing from limb emission spectra recorded from space by the Michelson Interferometer for Passive Atmospheric Sounding (MIPAS), J. Geophys. Res., 108, 4736, 2003.

von Savigny, C., Kaiser, J. W., Bovensmann, H., Burrows, J. P., McDonald, I. S., and Leblanc, T.: Spatial and temporal characterization of SCIAMACHY limb pointing errors during the first three years of the misson, Atmos. Chem. Phys., 5, 2593-2602, 2005 , http://www.atmos-chem-phys.net/5/2593/2005/. 